\title{
The Solution of Difference Equations by Continued Fractions.
}

\author{
By Prof. J. A. Strang.
}

(Received 15th August 1915. Read 12th November 1915).

I. The theorems which furnish in C.F. form the roots of a quadratic equation, and the similar process which leads to particular integrals of an ordinary differential equation of the second order, may be applied to certain types of difference equation. The types which suggest themselves for examination are

$$
u_{r} \cdot u_{r-s}=A_{r} \cdot u_{r}+B_{r} \cdot u_{r-s}+C_{r},
$$

the bilinear equation, and

$$
u_{r}=A_{r} \cdot u_{r-a}+B_{r} \cdot u_{r-2 s}
$$

a special form of the linear equation; the coefficients are functions of $r$, and $s$ is constant.

II. (a). To solve (1) by a terminating C.F. we have immediately

$$
\begin{aligned}
u_{r} & =\frac{B_{r} u_{r-s}+C_{r}}{u_{r-s}-A_{r}}=B_{r}+\frac{C_{r}+A_{r} B_{r}}{u_{r-s}-A_{r}}=B_{r}+\frac{D_{r}}{u_{r-s}-A_{r}} \text { say, } \\
& =B_{r}+\frac{D_{r}}{B_{r-s}-A_{r}}+\frac{D_{r-s}}{B_{r-2 s}-A_{r-s}}+\ldots+\frac{D_{r-(t-1) s}}{u_{r-k s}-A_{r-(k-1) s}}
\end{aligned}
$$

where $k$ is any integer. We may conveniently assume it, in most cases, to be the integral part of $r / s$.

$(\beta)$. The fact that the solution can be expressed as a C.F. suggests the equivalent form $u_{r}=\frac{a_{r} \cdot u+b_{r}}{c_{r} \cdot u+d_{r}}$, where $u$ is arbitrary, and the coefticients are functions of $r$.

Substituting in the relation

$$
u_{r}=\frac{B_{r} \cdot u_{r-s}+C_{r}}{u_{r-s}-A_{r}}
$$

we have $\frac{a_{r} \cdot u+b_{r}}{c_{r} \cdot u+d_{r}}=\frac{B_{r}\left(a_{r-s} \cdot u+b_{r-s}\right)+C_{r}\left(c_{r-s \cdot s} \cdot u+d_{r-s}\right)}{a_{r-s} \cdot u+b_{r-s}-A_{r}\left(c_{r-s} \cdot u+d_{r-s}\right)}$, 
which is identical if

$$
\begin{aligned}
& a_{r}=B_{r} \cdot a_{r-s}+C_{r \cdot} \cdot c_{r-s} \\
& b_{r}=B_{r \cdot} \cdot b_{r-s}+C_{r} \cdot d_{r-s} \\
& c_{r}=a_{r-s}-A_{r} \cdot c_{r-s} \\
& d_{r}=b_{r-s}-A_{r} \cdot d_{r-s}
\end{aligned}
$$

and

Eliminating $a$ from (1) and (3) we get

$$
c_{r+s}+\left(A_{r+s}-B_{r}\right) c_{r}-\left(A_{r} B_{r}+C_{r}\right) \cdot c_{r-s}=0,
$$

and $d_{r}$ satisfies the same equation.

Similarly we find that $a_{r}$ and $b_{r}$ are solutions of the equation

$$
C_{r} . a_{r+s}+\left(A_{r} . C_{r+s}-C_{r} . B_{r+s}\right) \cdot a_{r}-C_{r+s}\left(A_{r} B_{r}+C_{r}\right) a_{r-s}=0 .
$$

These equations are of the second type mentioned above, and it follows that the solution of the bilinear equation can be made to depend on that of either of two linear equations.

For example, we may solve (5) for $c_{r}$ and $d_{r}$, and then $a_{r}$ and $b_{r}$ are completely determined by (3) and (4). This result, although of some interest in itself as showing the close connection between the two types of equation, is not of much practical value except in a few cases, for, as a rule, the solutions of the linear equations will be incapable of expression in any very simple form, and may be more complex than the C.F. form of the solution of the bilinear equation. In some few instances a simple result is obtainable. This occurs, for example, when the coefficients of one of the linear equations are constant, or if any one coefficient vanishes identically, or if either of the linear equations belongs to one of the general forms mentioned below in IV. $(\alpha)$ and V.

$(\gamma)$. The question now arises as to the degree of generality of the solution we have obtained.

It is clear from the form of the equation (1) that the complete solution depends on $s$ different quantities. Thus the equation will determine $u_{r}$ for every value of $r$, provided we know the values of $u_{0}, u_{1}, \ldots u_{s-1}$. Nevertheless, the expression for $u_{r}$ can contain only one arbitrary constant; for in order to calculate $u_{r}$ for any given value of $r$, only one of the $s$ quantities is required, namely, $u_{\lambda}$, where $\lambda$ is the remainder when $r$ is divided by $s$. The C.F. form of the solution is therefore the most general one obtainable, provided that in any particular case the value of $k$ be chosen so that $u_{r-k s}$ is that difference upon which the value of $u_{r}$ depends. 
The number of partial quotients in the C.F. depends in fact on the particular $u$ which is to be regarded as arbitrary, and different C.F. are required to express the value of $u_{r}$, according as $r=k s+1, k s+2, \ldots$ or $k s+\overline{s-1}$.

Similarly, in the case of the equation $u_{r}=A_{r} \cdot u_{r-s}+B_{r} \cdot u_{r-2 s}$ the complete solution depends on $2 s$ arbitrary quantities. But the calculation of $u_{r}$ for any given value of $r$ depends on two only of these; therefore the expression for $u_{r}$ can contain only two arbitrary constants, and there may be, as before, $s$ different expressions for $u_{r}$, the particular one used depending on the form of $r$.

It only remains to note that the coefficients $a, b, c, d$ do not contain any arbitrary constant, since they are conditioned by the relations

$$
u_{0}=\frac{a_{0} \cdot u_{0}+b_{0}}{c_{0} \cdot u_{0}+d_{0}} \text { and } u_{s}=\frac{a_{s} \cdot u_{0}+b_{s}}{c_{s} \cdot u_{0}+d_{s}}=\frac{B_{s} \cdot u_{0}+C_{s}}{u_{0}-A_{s}},
$$

where, for the sake of simplicity, we have put $r-k s=0$.

( $\delta)$. Three exceptional cases remain to be discussed.

(1). If $A_{r} B_{r}+C_{r}=0$ identically, the bilinear equation reduces to two linear equations, $u_{r}-B_{r}=0$ and $u_{r-a}-A_{r}=0$, which need no further remark.

(2). The C.F. solution also fails if $A_{r}=B_{r-8}$ identically. We have then $\quad u_{r}, u_{r-s}=B_{r-s}, u_{r}+B_{r} \cdot u_{r-s}+C_{r}$,

which may be written

$$
\begin{aligned}
& \left(u_{r}-B_{r}\right)\left(u_{r-s}-B_{r-s}\right)=C_{r}+B_{r} \cdot B_{r-s} \\
& \text { or } \quad z_{r} \cdot z_{r-s}=D_{r}, \text { say. }
\end{aligned}
$$

Hence

$$
\begin{aligned}
z_{r} & =\frac{D_{r}}{z_{r-s}}=\frac{D_{r}}{D_{r-s}} \cdot z_{r-2 s} \\
& =\frac{D_{r}}{D_{r-s}} \cdot \frac{D_{r-2 s}}{D_{r-3 s}} \ldots \cdot \frac{D_{r-(2 m-2) s}}{D_{r-(2 m-1) s}} \cdot z_{r-2 m s}
\end{aligned}
$$

where $r-2 m s$ is less than $2 s$.

The solution now takes one or other of two forms, according as $r-2 m s$ is less than $s$ or not.

If $r-2 m s<s$, we simply replace $z_{r}$ and $z_{r-2 m s}$ by their values, and we have $u_{r}$, expressed in terms of $u_{r-2 m x}$, the latter being the arbitrary quantity in the solution. 
If $r-2 m s \equiv s$, we have $z_{r-2 m s}=\frac{D_{r-2 m s}}{z_{r-(2 m+1) s}}$, and the arbitrary quantity in the solution is $u_{r-(2 m+1) s}$. It is clear then that we get two different expressions for $u_{r}$, according as the integral part of $r / s$ is odd or even.

(3). In dealing with the third exception it is assumed that $r$ may have only integral values.

The exception is due to the fact that there are in general values of $r$ which satisfy the equation $C_{r}+A_{r}, B_{r}=0$, and one or more of the roots of this equation may be integral. With other roots we are not concerned. For any such value of $r$ we have

$$
\begin{aligned}
\left(u_{r}-B_{r}\right)\left(u_{r-r}-A_{r}\right) & =C_{r}+A_{r} B_{r} \\
& =0,
\end{aligned}
$$

so that either $u_{r}=B_{r}$ or $u_{r-s}=A_{r}$.

The point is that one or other of the two quantities $u_{r}, u_{r-s}$ has a definite value, depending on nothing arbitrary, and it is necessary therefore to examine more closely the form of the solution.

The net result is to introduce a kind of discontinuity into the solution. Instead of a single expression for $u_{r}$, valid for all values of the variable, we get a number of such expressions, each of which is applicable only for values of $r$ between two adjacent integral roots of the equation $C_{r}+A_{r} B_{r}=0$.

Take for example the equation $\left(u_{r}-1\right)\left(u_{r-1}-1\right)=r-3$,

$$
\begin{aligned}
& \text { or } \quad z_{r} \cdot z_{r-1}=r-3, \\
& \text { or } \quad z_{r}=\frac{r-3}{r-4}, z_{r-2} .
\end{aligned}
$$

If $r>3$, and $=2 n$ say, we get

$$
z_{r}=\frac{(2 n-3)(2 n-5)(2 n-7) \ldots 5.3}{(2 n-4)(2 n-6)} \frac{(2 n-8) \ldots 4.2}{\left(2 n-z_{4}\right.}=\frac{(2 n-3) \cdot(2 n-5) \ldots 5.3}{(2 n-4) \cdot(2 n-6) \ldots 4.2} \cdot \frac{1}{z_{3}}
$$

and if $r=2 n+1$,

$$
z_{r}=\frac{(2 n-2) \cdot(2 n-4) \ldots 4 \cdot 2}{(2 n-3) \cdot(2 n-5) \ldots 3 \cdot 1} \cdot z_{3} .
$$

Similarly, if $r<2$, we get, writing $r=-2 m$,

$$
z_{r}=\frac{(2 m+2) \cdot 2 m \cdot(2 m-2) \ldots 4 \cdot 2}{(2 m+1)(2 m-1)(2 m-3) \ldots 3 \cdot 1} z_{2}
$$


and if $r=-(2 m+1)$,

$$
z_{r}=\frac{(2 m+3)(2 m+1) \ldots 3}{(2 m+2) \cdot 2 m \ldots \ldots 2}\left(-\frac{1}{z_{2}}\right) .
$$

These, together with the condition that either $u_{3}$ or $u_{2}$ must be $=1$, constitute the complete solution, and enable us to calculate $u_{r}$ when any particular $u$ is known. If, for instance, we are given $z_{r}=8$, we have $z_{r}=\frac{4.2}{3.1} z_{3}=8, \therefore z_{3}=3, \therefore u_{3}=4$. Since $u_{3} \neq 1$ we must have $u_{2}=1$; and therefore all the $u$ 's are fixed in value. Similar reasoning applies to cases in which the equation $C_{r}+A_{r} . B_{r}=0$ has more than one integral root.

Consider, for instance, the equation $\left(u_{r}-r\right)\left(u_{r-1}-r^{2}\right)=r(r-4)$. The critical values of $r$ are 0 and 4 ; from the first we have $u_{0}=0$ or $u_{-1}=0$; and corresponding to the second value, $u_{4}=4$ or $u_{3}=16$.

Let us construct the scheme

$$
\ldots u_{-2} u_{-1}\left|u_{0} u_{1} u_{2} u_{3}\right| u_{4} u_{5} \ldots
$$

Suppose now that we are given $u_{-5}^{\prime}=k$. Starting from $u_{-5}$ we can calculate $u_{-1}$, and the value at which we arrive will not usually be 0 . Since $u_{-1} \neq 0$ we must have $u_{0}=0$, and this determines $u_{1}, u_{i 2}$, and $u_{3}$ completely. The value obtained for $u_{i j}$ will not in general be 16 , so we must put $u_{\downarrow}=4$; and all the other $u$ 's are determinate.

Again, if $u_{1}$ is given, we can determine $u_{0}$ and $u_{3}$ from the equation, and as a rule their calculated values will not be the critical values 0 and 16 found above. Hence we must put $u_{-1}=0$ and $u_{4}=4$, and all the remaining $u$ 's are again determinate. If the calculated value of $u_{0}$ were 0 , then $u_{-1}$ would remain arbitrary, and all the $u$ 's depending on it would be indeterminate to the extent of an arbitrary constant.

In general we should have a scheme such as

$$
\ldots u_{a-2} u_{\alpha-1}\left|u_{\alpha} u_{\alpha+1} \ldots u_{\beta-1}\right| u_{\beta} \ldots u_{\gamma-1} \mid u_{\gamma} \ldots
$$

where $\ldots \alpha, \beta, \gamma \ldots$ are the integral roots of $C_{r}+A_{r} . B_{r}=0$. From each compartment we can obtain from the equation an expression involving (as arbitrary constant) any one of the $u$ 's in that compartment. When any particular $u$ is given, we can 
determine from it the values of the end $u$ 's in that compartment, and proceed as in the particular examples given above.

It seems worthy of notice that if the equation $C_{r}+A_{r} B_{r}=0$ possesses integral roots, the specification of the value of any one $u$ may leave $u_{r}$ (for certain values of $r$ ) still indeterminate to the extent of an arbitrary constant.

(€). Finally, if the coefficients $A, B, C$ are constant, the equation admits of the trivial solution $u_{r}=z$, where $z$ is one of the roots of the quadratic equation

$$
z^{2}=(A+B) z+C
$$

These are obviously particular solutions only.

If the coefficients are not constant, we may obtain two particular solutions of the equation in the form of infinite C.F., provided the equation $C_{r}+A_{r} B_{r}=0$ has no integral roots. One of these is

$$
u_{r}=B_{r}+\frac{D_{r}}{B_{r-s}-A_{r}}+\frac{D_{r-s}}{B_{r-2 s}-A_{r-r}}+\ldots \text { to infinity. }
$$

The other is obtainable similarly from the equation

We find

$$
u_{r+s} . u_{r}=A_{r+s} . u_{r+s}+B_{r+s} . u_{r}+C_{r+s}
$$

$$
u_{r}=A_{r+\varepsilon}+\frac{D_{r+s}}{A_{r+2 s}-B_{r+s}}+\frac{D_{r+2 s}}{A_{r+3 s}-B_{r+2 s}}+\ldots \text { to infinity. }
$$

If the equation $C_{r}+A_{r} . B_{r}=0$ possesses integral roots, one or both of these particular solutions may be no longer obtainable.

It is evident that these two particular solutions become the roots of the quadratic when the coefficients are constant.

III. Examples. (1). $u_{r}, u_{r-1}+u_{r}=1$.

The C.F. solution is

$$
u_{r}=\frac{1}{1+u_{r-1}}=1+\frac{1}{1}+\frac{1}{1}+\ldots+\frac{1}{1+u_{0}}
$$

where there are $r$ partial quotients.

$$
\text { Putting } u_{r}=\frac{a_{r} u+b_{r}}{c_{r} u+d_{r}} \text { we find } \begin{aligned}
& a_{r}=c_{r-1} \\
& b_{r}=d_{r-1} \\
& c_{r}=a_{r-1}+c_{r-1} \\
& d_{r}=b_{r-1}+d_{r-1}
\end{aligned}
$$


so that

$$
u_{r}=\frac{x_{r \cdot} \cdot u+x_{r+1}}{x_{r+1} \cdot u+x_{r+2}}
$$

where

$$
x_{r+2}=x_{r+1}+x_{r} \text {, and } x_{1}=1, x_{2}=0 \text {. }
$$

That this furnishes a solution of the equation is easily verified.

By the method of IV. ( $\alpha$ ) we find $x_{r}=(-1)^{r+1}\left(A \beta^{r+1}+B \alpha^{r+1}\right)$ where $\alpha$ and $\beta$ are the roots of $x^{2}+x-1=0$, and

$$
A=\frac{2 \alpha-1}{\alpha-\beta}, B=\frac{2 \beta-1}{\beta-\alpha} .
$$

Wo may easily verify that on putting $u=\alpha$, we get $u_{r}=\alpha$. The infinite C.F. are

$$
\begin{aligned}
& u=1+\frac{1}{1}+\frac{1}{1}+\ldots \text { to infinity }=\frac{\sqrt{5}-1}{2}, \\
& u=-1-\frac{1}{1}-\frac{1}{1} \ldots \text { to infinity }=-\frac{\sqrt{5}-1}{2} .
\end{aligned}
$$

(2) $u_{x+1} \cdot u_{x}+x \cdot u_{x}=x^{2}$.

We obtain easily

$$
u_{x}=-(x-1)+\frac{(x-1)^{2}}{-(x-2)}+\frac{(x-2)^{2}}{-(x-3)}+\ldots+\frac{1^{2}}{\bar{u}_{1}} .
$$

The linear equations for $a, b, c, d$ both reduce to

$$
u_{r+1}=r^{2} \cdot u_{r-1}-r \cdot u_{r}
$$

but the general solution of this linear equation is not so simple.

The C.F. may be transformed into a series without much difficulty. The result is

$$
\begin{aligned}
\frac{u_{x}}{1-x}=1-(x-1) & +(x-1)(x-2)+\ldots \\
& +(-1)^{x-1} \frac{(x-1) !}{2 !}+\frac{(-1)^{x} u_{1}(x-1) !}{1+u_{1}}
\end{aligned}
$$

The same thing is possible in other cases, but in every case the trouble of transformation is considerable; verification, when possible at all, is no longer easy; the vexed questions of equivalence and convergence arise, particularly when C.F. and series are infinite; and no advantage is gained by the transformation. 
IV. We now proceed to consider the equation

$$
u_{r}=A_{r} \cdot u_{r-s}+B_{r} \cdot u_{r-2 s} \text {. }
$$

$(x)$. When the coefficients are constant, the complete solution is easily effected by the aid of a recurring series.

Take, for example, the equation $x_{r}+a \cdot x_{r-1}+b \cdot x_{r-2}=0$.

Writing $\quad y=x_{0}+x_{1} z+x_{2} z^{2}+\ldots+x_{r} z^{r}+\ldots$,

we have

and

Hence $y\left(1+a z+b z^{2}\right)=x_{0}+\left(x_{1}+a x\right.$
if the coefficients satisfy the given equation.

$$
\begin{gathered}
a z y=a x_{0} \cdot z+a x_{1} \cdot x^{2}+\ldots+a x_{r-1} \cdot z^{r}+\ldots \\
b z^{2} y=b x_{0} \cdot z^{2}+\ldots+b x_{r-2} \cdot z^{r}+\ldots .
\end{gathered}
$$

Hence

$$
y=\frac{x_{0}+\left(x_{1}+a x_{0}\right) z}{1+a z+b z^{2}},
$$

and to find $x_{r}$ we need only expand in ascending powers of $z$, and equate $x_{r}$ to the coefficient of $z^{r}$.

The equation

$$
f(r) \cdot x_{r}+p \cdot f(r-1) \cdot x_{r-1}+q \cdot f(r-2) \cdot x_{r-2}+\ldots=a \cdot \lambda^{r}
$$

is immediately soluble by this method, on putting $z_{r}=x_{r}, f(r)$ ( $a$ and $\lambda$ being constants).

$(\beta)$. The above method may be extended to a few cases in which the coefficients are not constant.

Take, for instance, $x_{r}+p(r+1) x_{r+1}+q(r+2)(r+1) x_{r+2}=0$. As before, we have $y=x_{0}+x_{1} z+x_{2} z^{\prime \prime}+\ldots+x_{r} \cdot z^{r}+\ldots$

$$
\begin{aligned}
& p . D y=p x_{1}+2 p x_{1} z+\ldots+p(r+1) x_{r+1} \cdot z^{r}+\ldots \\
& q . D^{2} y=2 q \cdot x_{2}+\ldots+q(r+2)(r+1) x_{r+2} \cdot z^{r}+\ldots
\end{aligned}
$$

so that on adding we obtain

$$
\left(1+p . D+q . D^{2}\right) y=0 .
$$

If the roots of the quadratic $1+\eta \cdot \xi+q \cdot \xi^{2}=0$ are unequal, $(\lambda, \mu)$ say, we have as the general solution

$$
y=A e^{\lambda z}+B e^{\mu z},
$$

and therefore

$$
x_{r}=\frac{1}{r !}\left(A \lambda^{r}+B \mu^{r}\right),
$$

where, of course, $A+B=x_{0}$, and $A \lambda+B \mu=x_{1}$.

If the roots are equal, the solution of the differential equation will be

$$
y=(A+B z) e^{\lambda z} \text {, }
$$


and therefore

$$
x_{r}=\frac{\lambda^{r-1}}{r !}(A \lambda+B r)
$$

where $A=x_{0}$, and $A \lambda+B=x_{1}$.

In either case these expressions supply the general solution of the difference equation, the quantities $x_{0}$ and $x_{1}$ being arbitrary; and verification by substitution is easy.

This method is of very limited application. It evidently is only applicable when the differential equation which results is capable of solution in comparatively simple form, and when $y$ can be expanded in a series of the assumed form. The failure of the method is easily illustrated.

Suppose that $\quad x_{n}=n x_{n-1}+x_{n-1 \cdot}$. We have then

$$
\begin{aligned}
y & =x_{0}+x_{1} z+\ldots+x_{n} \cdot z^{n} \ldots \\
-z D(z y) & =-x_{0} z \ldots \ldots-n \cdot x_{n-1} \cdot z^{n} \ldots \\
-z^{2} y & =-x_{0} z^{2} \ldots \ldots-x_{n-2} \cdot z^{n} \ldots,
\end{aligned}
$$

so that if the coefficients obey the assumed law

$$
\left(1-z^{2}\right) y-z D(z y)=x_{0}+\left(x_{1}-x_{0}\right) z,
$$

which simplifies to

$$
\begin{gathered}
D y+\left(1+\frac{1}{z}-\frac{1}{z^{2}}\right) y=-\frac{x_{0}}{z^{2}}+\frac{x_{0}-z_{1}}{z} \\
\text { or } \quad y z e^{z+\frac{1}{z}}=K+\int\left(x_{0}-x_{1}-\frac{x_{0}}{z}\right) e^{z+\frac{1}{z}} \cdot d z .
\end{gathered}
$$

It is evident that even if the quadrature could be performed, $y$ is not expressible in a series of the assumed form; and the reason is obvious, for $x_{n}$ is comparable with (and greater than) $n$ !, so that the assumed series could not converge for any value of $z$ whatever, except $z=0$. Nor can we surmount the difficulty by assuming for $y$ a series infinite both ways, and neglecting all but formal equivalence; the integral on the right hand side would then disappear, but the determination of $K$ raises new difficulties, apart, of course, from the fact that we have no right at all to differentiate such a series.

$(\gamma)$. It remains to show how the general solution may be obtained by the use of C.F. 
We have $u_{r}=A_{r} \cdot u_{r-s}+B_{r} \cdot u_{r-9 s}$.

$$
\begin{aligned}
\therefore \frac{u_{r}}{u_{r-s}} & =A_{r}+\frac{B_{r}}{\frac{u_{r-s}}{u_{r-2 s}}} \\
& =A_{r}+\frac{B_{r}}{A_{r-s}}+\frac{B_{r-*}}{A_{r-2 s}}+\cdots+\frac{B_{r-(k-2) s}}{\frac{u_{r-(k-1) s}}{u_{r-k s}}}=C_{r} \text {, say. }
\end{aligned}
$$

Similarly $\frac{u_{r-s}}{u_{r-9 s}}=C_{r-s}, \frac{u_{r-2 s}}{u_{r-3 s}}=C_{r-9 s}$, and so on.

Hence

$$
\begin{aligned}
u_{r} & =\frac{u_{r}}{u_{r-s}} \cdot \frac{u_{r-s}}{u_{r-2 s}} \ldots \ldots \frac{u_{r-(k-1) s}}{u_{r-k s}} \cdot u_{r-k s} \\
& =C_{r} \cdot C_{r-s} . C_{r-2 s} \ldots \ldots C_{r-(k-1) s} \cdot u_{r-k s} .
\end{aligned}
$$

This is the general solution required. It contains two arbitrary quantities, namely, $u_{r-(k-1) s}$ and $u_{r-i s}$. The ratio of these is the last partial quotient in each of the $C^{\prime \prime}$ s.

We can at once verify the solution by substitution in the original equation, for we find

$$
C_{r} . C_{r-s} \ldots u_{r-2 k s}=A_{r} . C_{r-s} . C_{r-2 s} \ldots u_{r-k s}+B_{r} . C_{r-2 s} \ldots u_{r-k s},
$$

which is true if

$$
C_{r} . C_{r-s}=A_{r} . C_{r-s}+B_{r}
$$

i.e. if $\quad C_{r}=A_{r}+\frac{B_{r}}{C_{r-s}}$, which is true.

(8). Those cases in which either or both of the coefficients vanish for some particular value or values of $r$ nay be best illustrated by examples.

(1). $u_{r}=(r-6) \cdot u_{r-1}+r^{2} \cdot u_{r-3} \cdot$

$$
\text { When } r=6 \text { we get } u_{6}=6^{2} . u_{\Downarrow} \text {, so that if } r>6 \text {, }
$$

$$
\begin{aligned}
\frac{u_{r}}{u_{r-1}}=r-6+\frac{r^{2}}{\frac{u_{r-1}}{u_{r-2}}} & =r-6+\frac{r^{2}}{r-7}+\frac{(r-1)^{2}}{r-8}+\ldots+\frac{8^{2}}{1}+\frac{7^{2}}{\frac{u_{6}}{u_{5}}} \\
& =r-6+\frac{r^{2}}{r-7}+\frac{(r-1)^{2}}{7-8}+\ldots+\frac{8^{2}}{1}+\frac{7^{2}}{6^{2}} \cdot \frac{u_{5}}{u_{4}} .
\end{aligned}
$$

But

$$
\frac{u_{5}}{u_{4}}=-1+\frac{5^{2}}{-2}+\frac{4^{2}}{-3}+\frac{3^{2}}{-4}+\frac{2^{2}}{\frac{u}{u_{0}}} \text {, }
$$


so that evidently there is no failure of the solution. The only effect is a slight disturbance of the form of each C.F. The same happens in general if $A_{r}=0$ for any particular value of $r$.

(2). As an instance of a value of $r$ which makes $B_{r}=0$, take the equation

$$
u_{r}=r^{\prime} \cdot u_{r-1}+(r-6) u_{r-2} \text {. }
$$

Here we have $u_{6}=6^{2} . u_{5}$, so that if $r>6$,

and therefore

$$
\begin{aligned}
\frac{u_{r}}{u_{r-1}} & =r^{2}+\frac{r-6}{(r-1)^{2}}+\frac{r-7}{(r-2)^{2}}+\ldots+\frac{3}{8^{2}}+\frac{2}{7^{2}}+\frac{1}{\frac{u_{6}}{u_{5}}} \\
& =r^{2}+\frac{r-6}{(r-1)^{2}}+\frac{r-7}{(r-2)^{2}}+\ldots+\frac{3}{8^{2}}+\frac{2}{7^{2}}+\frac{1}{6^{2}}=C_{r},
\end{aligned}
$$

$$
u_{r}=C_{r} . C_{r-1} . C_{r-2} \ldots C_{6} \cdot u_{5}
$$

where none of the $C$ 's contains an arbitrary quantity, so that $u_{r}$ depends only on $u_{5}$, so long as $r>5$.

If, however, we regard $u_{1}$ and $u_{0}$ as arbitrary, and express others in terms of these, we have

$$
u_{5}=\frac{u_{5}}{u_{4}} \cdot \frac{u_{4}}{u_{3}} \cdot \frac{u_{3}}{u_{3}} \cdot \frac{u_{2}}{u_{1}} \cdot \frac{u_{1}}{u_{0}} \cdot u_{0}
$$

and since each of these ratios depends on $u_{1} / u_{0}$, it follows that $u_{5}$ alone introduces two arbitrary quantities into the expression for $u_{r}$

The exception, so far as it exists, consists in the fact that if either $u_{5}$ or $u_{6}$ be arbitrary, $u_{r}$ depends only on one quantity, and not on two.

(3). Suppose now that $A_{r}=0$ and $B_{r}=0$ for the same value of $r$, as in the equation $u_{r}=(r-6)\left(u_{r-1}+u_{r-2}\right)$, so that $u_{6}=0$.

$$
\begin{aligned}
& \text { We find } \begin{aligned}
\frac{u_{r}}{u_{r-1}} & =(r-6)+\frac{r-6}{r-5}+\frac{r-5}{r-4}+\ldots+\frac{4}{3}+\frac{3}{2}+\frac{2 u_{6}}{u_{r}} \\
& =(r-6)+\frac{r-6}{r-5}+\frac{r-5}{r-4}+\ldots+\frac{4}{3}+\frac{3}{2}, \text { since } u_{6}=0, \\
& =C_{r} \text { say. }
\end{aligned} \\
& \begin{aligned}
\therefore \quad u_{r}=C_{r} . C_{r-1} \cdot C_{r-2} \ldots C_{8} \cdot u_{7} \\
=C_{r} . C_{r-1} \cdot C_{r-2} \ldots C_{8} \cdot u_{3}, \quad \text { since } u_{7}=(7-6)\left(u_{6}+u_{5}\right) \\
=u_{5},
\end{aligned}
\end{aligned}
$$

and as before,

$$
u_{5}=\frac{u_{5}}{u_{4}} \cdot \frac{u_{4}}{u_{3}} \cdot \frac{u_{3}}{u_{3}} \cdot \frac{u_{2}}{u_{1}} \cdot \frac{u_{1}}{u_{0}} \cdot u_{0}
$$

Here again the exception is of the same nature. 
(€). Since the difference equation is linear, it follows that the sum of any number of particular solutions, each multiplied by an arbitrary constant, is itself a solution. It is interesting to note that the above expression may be reduced to this form.

For suppose that $C_{r}=\frac{a_{r} \lambda+b_{r}}{c_{r} \lambda+d_{r}}$, where $\lambda=\frac{u_{x}}{u}$, and we put $r-k s=0$ for convenience.

Substituting in the last equation of IV. $(\gamma)$, we find

$$
\frac{a_{r} \lambda+b_{r}}{c_{r} \lambda+d_{r}}=A_{r}+\frac{B_{r}\left(c_{r-s} \cdot \lambda+d_{r-s}\right)}{a_{r-s} \cdot \lambda+b_{r-s}},
$$

which is an identical relation provided

$$
\begin{aligned}
a_{r} & =A_{r} \cdot a_{r-s}+b_{r} \cdot c_{r-s} \\
b_{r} & =A_{r} \cdot b_{r-s}+B_{r} \cdot d_{r-s} \\
c_{r} & =a_{r-s} \\
d_{r} & =b_{r-s} .
\end{aligned}
$$

From these equations it follows that $a_{r}$ and $b_{r}$ are particular solutions of the given difference equation, and from the last two, that

so that

$$
\begin{aligned}
& C_{r}=\frac{a_{r} \cdot \lambda+b_{r}}{a_{r-s} \cdot \lambda+b_{r-s}}, \\
u_{r}= & C_{r} \cdot C_{r-s} \ldots C_{r-(k-1) s} u_{r-k s} \\
= & \left(a_{r} \lambda+b_{r}\right) u_{0}, \quad \text { when we take account of the } \\
= & a_{r_{-}} u_{s}+b_{r} u_{0}, \quad \text { initial values of } a \text { and } b,
\end{aligned}
$$

the form required, since $u_{0}$ and $u_{s}$ are arbitrary.

V. (1). The general solution of

$$
x_{r-2}+p(r-1) x_{r-1}+q(r-1) r \cdot x_{r}=0
$$

has been obtained in IV. $(\beta)$.

It is

$$
x_{r}=\frac{1}{r !}\left[\frac{\left(\mu x_{0}-x_{1}\right) \lambda^{r}-\left(\lambda x_{0}-x_{1}\right) \mu^{r}}{\mu-\lambda}\right]
$$

where $x_{0}$ and $x_{1}$ are arbitrary, and $\lambda, \mu$ are the roots of $1+p \xi+q \xi^{2}=0$.

The solution by C.F. is as follows :

The equation may be written

$$
\begin{gathered}
-r \frac{x_{r}}{x_{r-1}}=\frac{p}{q}+\frac{1}{q(r-1) \frac{x_{r-1}}{x_{r-2}}} \\
\text { or } z_{r}=\frac{p}{q}-\frac{1}{q \cdot z_{r-1}} \quad \text { where } z=-r \cdot \frac{x_{r}}{x_{r-1}},
\end{gathered}
$$


This is a bilinear equation with constant coefficients, whose solution is

$$
z_{r}=\frac{a_{r} \cdot z_{1}+b_{r}}{a_{r-1} \cdot z_{1}+b_{r-1}}
$$

where $a_{r}=\frac{1}{\beta-\alpha}\left[\frac{\beta}{\alpha^{r-1}}-\frac{\alpha}{\beta^{r-1}}\right]$ and $b_{r}=\frac{1}{\beta-\alpha}\left[\frac{1}{\beta^{r-1}}-\frac{1}{\alpha^{r-1}}\right]$,

$\alpha$ and $\beta$ being the roots of $\eta^{2}-p \eta+q=0$.

$$
\begin{aligned}
& \text { Hence } \begin{aligned}
z_{r} \cdot z_{r-1} \cdot z_{r-2} \ldots z_{2} \cdot z_{1} & =\frac{a_{r} \cdot z_{1}+b_{r}}{a_{r-1} \cdot z_{1}+b_{r-1}} \ldots \frac{a_{2} z_{1}+b_{2}}{a_{1} z_{1}+b_{1}} \cdot z_{1} \\
& =a_{r} z_{1}+b_{r},
\end{aligned} \\
& \text { i.e. }(-1)^{r}(r !) \frac{x_{r}}{x_{0}}=-a_{r} \cdot \frac{x_{1}}{x_{0}}+b_{r}, \\
& \text { or } x_{r}=\frac{(-1)^{r-1}}{r !(\beta-\alpha)}\left[\frac{\beta x_{1}+x_{0}}{a^{r-1}}-\frac{\alpha x_{1}+x_{0}}{\beta^{r-1}}\right]
\end{aligned}
$$

where $\alpha, \beta$ are the roots of $\eta^{2}-p \eta+q=0$,

$$
=\frac{1}{r !(\mu-\lambda)}\left[\left(\mu x_{0}-x_{1}\right) \lambda^{r}-\left(\lambda x_{0}-x_{1}\right) \mu^{r}\right]
$$

where $\lambda, \mu$ are the roots of $1+p \xi+q \xi^{2}=0$,

which is the previous form.

If, in solving the equation for $z_{r}$, we proceed to form an infinite C.F., we find

$$
\begin{aligned}
z_{r} & =\frac{p}{q}-\frac{1}{p}-\frac{1}{p / q}-\frac{1}{p} \ldots \text { to infinity, } \\
& =\alpha, \quad \text { where } \alpha \text { is a root of } 1-p \alpha+q \alpha^{2}=0, \\
& =-\lambda, \text { where } \lambda \text { is a root of } 1+p \lambda+q \lambda^{2}=0 . \\
\therefore \quad \frac{x_{r}}{x_{r-1}} & =\frac{\lambda}{r},
\end{aligned}
$$

$$
\text { and } \begin{aligned}
\therefore \quad x_{r} & =\frac{x_{r}}{x_{r-1}} \cdot \frac{x_{r-1}}{x_{r-2}} \ldots \frac{x_{1}}{x_{0}} \cdot x_{0} \\
& =\frac{\lambda^{r} \cdot x_{0}}{r !},
\end{aligned}
$$

which is a particular solution derivable from the general expression by putting $x_{1}=\lambda x_{0}$. 
We may evidently obtain by an exactly similar process the solution of the equation

$$
x_{r-2}+p \cdot \phi(r-1) x_{r-1}+q \cdot \phi(r-1) \cdot x_{r}=0
$$

where $\phi(r)$ is any function of $r$.

The result is then

$$
x_{r}=\frac{P}{\mu-\lambda}\left[\left(\mu x_{0}-x_{1}\right) \lambda^{r}-\left(\lambda x_{0}-x_{1}\right) \mu^{r}\right]
$$

where $\quad \frac{1}{P}=\phi(r) \cdot \phi(r-1) \cdot \phi(r-2) \ldots \phi(2) \cdot \phi(1)$.

The expression takes a slightly different but easily obtained form if the equation $\phi(r)=0$ has one or more integral roots.

(2). A difference equation of considerable importance in mathematical "physics is that satisfied by the coefficients of Legendre, namely,

$$
n \cdot P_{n}=(2 n-1) \mu, P_{n-1}-(n-1) \cdot P_{n-2} .
$$

From it we have

$$
\begin{aligned}
\frac{P_{n}}{P_{n-1}} & =\frac{(2 n-1) \mu}{n}-\frac{(n-1) / n}{P_{n-1} / P_{n-3}} \\
& =\frac{(2 n-1) \mu}{n}-\frac{(n-1) / n}{(2 n-3) \mu /(n-1)}-\frac{(n-2) /(n-1)}{(2 n-5) \mu /(n-2)}-\ldots-\frac{2 / 3}{P_{2} / P_{1}}
\end{aligned}
$$

which may also be written

$$
n \cdot \frac{P_{n}}{P_{n-1}}=(2 n-1) \mu-\frac{(n-1)^{2}}{(2 n-3) \mu}-\frac{(n-2)^{2}}{(2 n-5) \mu}-\ldots-\frac{3^{2}}{5 \mu}-\frac{2}{P_{2} / P_{1}} .
$$

But $\begin{aligned} & P_{2}=\frac{1}{2}\left(3 \mu^{2}-1\right) \\ & P_{1}=\mu\end{aligned}$

and $\therefore \quad \frac{P_{2}}{P_{1}}=\frac{3 \mu}{2}-\frac{1}{2 \mu}$. 


\section{5}

Hence

$$
\begin{aligned}
n \cdot \frac{P_{n}}{P_{n-1}} & =(2 n-1) \mu-\frac{(n-1)^{2}}{(2 n-3) \mu}-\frac{(n-2)^{2}}{(2 n-5) \mu}-\ldots-\frac{3^{2}}{5 \mu}-\frac{2^{2}}{3 \mu}-\frac{1^{2}}{\mu} . \\
& =C_{n}, \text { say, }
\end{aligned}
$$

and $\quad \therefore n ! \cdot \frac{P_{n}}{P_{1}}=C_{n} \cdot C_{n-1} \ldots C_{2}$

and since

$$
\begin{aligned}
& P_{1}=\mu=C_{1}, \\
& P_{n}=\frac{1}{n !} \cdot \prod_{1}^{n} C
\end{aligned}
$$

an expression for $P_{n}$ which is, $I$ believe, new. 